\title{
An online throughput-competitive algorithm for multicast routing and admission control ${ }^{\text {嵓 }}$
}

\author{
Ashish Goel $^{\mathrm{a}, *, 1}$, Monika R. Henzinger ${ }^{\mathrm{b}, 2}$, Serge Plotkin ${ }^{\mathrm{c}, 3}$ \\ a Departments of Management Science and Engineering and (by courtesy) Computer Science, \\ Stanford, CA 94305, USA \\ b Google Inc., 2400 Bayshore Parkway, Mountain View, CA 94043, USA \\ c Department of Computer Science, Stanford, CA 94305, USA
}

Received 9 January 2001

Available online 8 December 2004

\begin{abstract}
We present the first polylog-competitive online algorithm for the general multicast admission control and routing problem in the throughput model. The ratio of the number of requests accepted by the optimum offline algorithm to the expected number of requests accepted by our algorithm is $O((\log n+\log \log \mathcal{M})(\log n+\log \mathcal{M}) \log n)$, where $\mathcal{M}$ is the number of multicast groups and $n$ is the number of nodes in the graph. We show that this is close to optimum by presenting an $\Omega(\log n \log \mathcal{M})$ lower bound on this ratio for any randomized online algorithm against an oblivious adversary, when $\mathcal{M}$ is much larger than the link capacities. Our lower bound applies even in the restricted case where the link capacities are much larger than bandwidth requested by a single
\end{abstract}

\footnotetext{
A A preliminary version of these results appeared in the 9th ACM-SIAM Symposium on Discrete Algorithms, 1998.

* Corresponding author.

E-mail addresses: ashishg@stanford.edu (A. Goel), monika@google.com (M.R. Henzinger), plotkin@cs.stanford.edu (S. Plotkin).

1 Research supported by an NSF Career award, an Alfred P. Sloan Fellowship, and by Serge Plotkin's ARO and NSF Grants.

2 The author was at the Digital Systems Research Center, Digital Equipment Corporation when this research was done.

3 Research supported by ARO Grant DAAH04-95-1-0121, NSF Grants CCR9304971/CCR9307045, and by a Terman Fellowship.
}

0196-6774/\$ - see front matter ㄷ 2004 Elsevier Inc. All rights reserved. doi:10.1016/j.jalgor.2004.11.001 
multicast. We also present a simple proof showing that it is impossible to be competitive against an adaptive online adversary.

As in the previous online routing algorithms, our algorithm uses edge-costs when deciding on which is the best path to use. In contrast to the previous competitive algorithms in the throughput model, our cost is not a direct function of the edge load. The new cost definition allows us to decouple the effects of routing and admission decisions of different multicast groups.

(c) 2004 Elsevier Inc. All rights reserved.

Keywords: Multicast; Routing; Admission control; Online

\section{Introduction}

We study the problem of online multicast admission control and routing in a capacitated network, where both requests to form new groups and requests to join existing multicast groups arrive in an online fashion. Each multicast group requires a certain amount of reserved bandwidth which has to be allocated along a tree spanning the nodes participating in the multicast group. In the general case, a multicast request specifies the user (endpoint) and the multicast group that this user wants to participate in. The router should either reject the request or accept it and allocate bandwidth along a path connecting the new endpoint with the already existing tree for this group. The total allocated bandwidth on a link should not exceed the capacity of the link.

In this paper we present the first polylog-competitive algorithm for the general multicast problem. Our algorithm is randomized, as it must be since we also show that it is impossible for any deterministic algorithm to achieve a polylogarithmic competitive ratio. The ratio of the number of requests accepted by the optimum offline algorithm to the expected number of requests accepted by our algorithm is $O((\log n+\log \log \mathcal{M})(\log n+\log \mathcal{M}) \log n)$, where $\mathcal{M}$ is the number of multicast groups and $n$ is the number of nodes in the graph (i.e., the network). If each vertex is allowed to serve at most one multicast group, the competitive ratio simplifies to $O\left(\log ^{3} n\right)$.

Like earlier work on this problem, our algorithm also requires that the maximum bandwidth requirement of a multicast group be smaller than the minimum capacity of an edge by a factor that is at least logarithmic in the network size.

\section{Unicast routing}

Routing and admission control problems in the online setting have been extensively studied. Two related performance measures have been considered. In the congestion model, the algorithm is required to accept all of the requests, and the goal is to minimize the maximum edge congestion (utilization). Here the congestion is allowed to exceed $100 \%$. In the throughput model the algorithm is allowed to reject some of the requests and is not allowed to exceed $100 \%$ congestion on any link. The goal is to maximize the total bandwidth-duration product for all the accepted (routed) requests.

The first competitive algorithm in the congestion model for general topology networks was presented in [1]. The competitive ratio of this algorithm is $O(\log n)$, where $n$ is the 
number of nodes in the network. The first competitive algorithm in the throughput model was given in [10] for the case of a single-link network and extended in [9] for a line network. A competitive algorithm for general topology networks in the throughput model was presented in [4]. This algorithm achieves $O(\log n T)$ competitive ratio, where $T$ is the maximum duration (holding time) of a virtual circuit. The competitive ratio improves to $O(\log n)$ for the Permanent Virtual Circuits case, i.e., circuits with infinite holding times. The algorithm assumes that each circuit specifies its holding time upon arrival. It is impossible to achieve polylogarithmic competitive ratio if the holding times become known only upon termination of the circuit [6,20]. Further, the throughput-competitive algorithms mentioned above assume that the bandwidth requested by any one connection is smaller than the capacity of any link by a factor which is at least logarithmic in the network size.

Routing in a probabilistic model where there are assumptions on the distribution of call arrival times and source-destination pairs was considered in [16]. Their results were based on the edge-independence assumption, which states that the random variables describing instantaneous load on edges are independent. This assumption is not satisfied in general, and hence their results are incomparable to the ones presented in this paper. The randomized model without the independence assumption was considered in [14]. In the case where the durations are exponentially distributed and the arrivals are Poisson with unknown rates, their algorithm achieves a $(1+\varepsilon)$ competitive ratio, where $\varepsilon$ depends on the ratio of the minimum capacity to maximum bandwidth of a single virtual circuit. Both [4] and [14] assume at least logarithmic ratio between maximum virtual circuit bandwidth and minimum link capacity. Similar results without this assumption were developed for special network topologies (see, e.g., [18]).

All of the above algorithms are related to the (offline) combinatorial approximation algorithms for multicommodity flow [15,17,19,21]. As in these algorithms, the basic idea is to assign each edge a cost that is exponential in the congestion on this edge, and try to route along short paths with respect to this cost.

\section{Multicast routing}

The techniques in the above mentioned papers can be used to solve several restricted multicast problems in the throughput model. In particular, [4] shows that if the participants in a single multicast group arrive together ("batch arrivals"), and the accept/reject decision is for the whole multicast group, it is possible to achieve an $O(\log n)$ competitive ratio. Roughly, the idea is to route the multicast request along a minimum-cost Steiner tree; this is analogous to routing along a shortest path for unicast. The case where we keep the restriction of batch arrivals, but allow rejection of some of the group members and acceptance of others can be solved by replacing an approximation algorithm for Steiner tree with an approximation algorithm for the k-MST problem. The first polylogarithmic and constant factor approximations for this problem were presented in [2,8], and a 3-approximation algorithm was given in [11]. These k-MST algorithms are relatively slow and use a polynomial number of calls to an approximation algorithm for the prize-collecting traveling salesman problem [13]. The techniques developed in this paper allow the online multicast algorithm to make a single call to the prize-collecting traveling salesman algorithm for each batch arrival. 
Awerbuch and Singh [5] showed how to combine the "winner-picking" technique [3] with the techniques in [4] to achieve a polylog competitive ratio for the case where members of each multicast group arrive sequentially, i.e., the size and membership of the group is unknown upon its creation. Their algorithm can deal only with the non-interleaved case, i.e., when all the members of a particular multicast group arrive before a new group can be created.

The algorithm in [5] is not applicable in the general case, where the arrivals of requests belonging to different multicast groups are interleaved. The main problem is that this algorithm depends on the fact that, at every instance, the algorithm is dealing with the construction of only a single multicast tree and all accept/reject decisions with respect to all existing multicast groups are already known. As in [4], the algorithm in [5] uses edge-costs that are exponential in the current link load. One of our contributions is a new definition of edge-costs that are independent of the specific accept/reject decisions made with respect to each multicast group. This decoupling between multicast groups is what allows us to generalize the algorithm in [5] and to combine it with techniques in [4] to achieve a polylog competitive ratio for the general multicast problem.

\section{Lower bounds}

A natural question to ask is if it is possible to make the competitive ratio independent of $\mathcal{M}$, the number of multicast groups. We address this issue by showing a lower bound of $\Omega(\log \mathcal{M} \log n)$ when $\mathcal{M}$ is much larger than the link capacities. This is the first bound for this problem that is stronger than $\Omega(\log n)$ and that works even if we require that the bandwidth requested by each multicast is significantly smaller than bandwidth of a single link. A similar lower bound for the case where a multicast is allowed to request a constant fraction of a link bandwidth was shown in [3]. For the case where a multicast can request bandwidth equal to bandwidth of a single link, a polynomial lower bound was shown in [7].

It is interesting to note that the algorithm presented in this paper works even against a semi-oblivious adversary, i.e., the adversary is allowed to look at the tree used by the online algorithm to service a multicast group only after all the requests for that group have been processed. ${ }^{4}$ We show that against an adaptive online adversary, any randomized online algorithm must have a competitive ratio of $\Omega\left(\min \left(n, \frac{\mathcal{M}}{u}\right)\right)$. As a corollary, any deterministic algorithm must also have a competitive ratio of $\Omega\left(\min \left(n, \frac{\mathcal{M}}{u}\right)\right)$.

Previous papers on online multicast routing and admission control often ignored the issue of computational complexity. In particular, the algorithm in [5] assumes an NP-hard computation at each routing decision. We show that it is possible to use the special properties of the prize-collecting Steiner tree algorithm in [13] to implement each step of our algorithm in polynomial time; the same technique also allows us to give a polynomial time, $O(\log n)$-competitive algorithm for selective batch arrival multicast requests (see Appendix A.3 for a description of the problem).

In Section 2 we introduce the model and the terminology. Section 3 describes the algorithm, and Section 5 presents the proof of the competitive ratio. The algorithm as described

4 A semi-oblivious adversary is at least as powerful as an oblivious adversary. 
in Section 3 may violate capacity constraints on edges. In Section 6 we show that the above algorithm can be slightly modified to guarantee that capacity constraints are never violated. In Section 6.1 we remark that several of the assumptions made in the earlier sections can be relaxed. Lower bounds are presented in Section 7. Appendix A explains how to implement each decision step of our online algorithm in polynomial time and also addresses the selective batch arrival problem. A general introduction to competitive analysis can be found in the work of Sleator and Tarjan [22].

\section{Model and definitions}

We model the network as a capacitated graph with $n$ nodes and $m$ edges. Edge $e$ has capacity $u_{e}$. Requests are of two types-for creating a new group or joining an existing group.

The $i$ th request to create a multicast group specifies the source $s_{i}$ and the bandwidth requirement $r_{i}$ of this multicast group. A multicast algorithm does not need to explicitly accept or reject this request since no bandwidth reservation is required for the new group at this stage. The online algorithm maintains a tree $T_{i}$ for group $i$; initially the tree consists of the single node $s_{i}$.

A "join" request specifies the group $i$ and the node $v$ that wants to join this group. The multicast routing and admission algorithm can either reject a join request or accept it and reserve bandwidth $r_{i}$ along some path from $v$ to $T_{i}$; this path is also added to $T_{i}$. The total reserved bandwidth on any link must not exceed the capacity of that link.

For simplicity, we will assume that all edges have the same capacity $u$ and all groups have the same bandwidth requirement $r_{i}=1$. We also assume that the number of multicast groups $\mathcal{M}$ is known in advance. The issue of removing these assumptions is deferred to Section 6.1.

We also assume that $u \geqslant \log \mu$, where $\mu$ is a parameter that is polynomial in $n$ and $\mathcal{M}$, and will be defined later. ${ }^{5}$ We assume that multicast groups, once established, never leave.

\section{The algorithm}

The online algorithm can be viewed as consisting of $L=\log n+\log \mathcal{M}$ "virtual" algorithms for each one of the $\mathcal{M}$ multicast groups. We call these algorithms virtual because the routing and accept/reject decisions of these algorithms are not implemented. Instead, they only modify internal data structures and, in particular, the cost associated with each edge. The description of the cost computation is deferred to Section 4. For now, it is sufficient to assume that each edge has an associated cost that is deterministic, depends only on the input sequence of requests, and is monotonically non-decreasing in time. For a set of edges $S$, we use $\operatorname{Cost}(S)$ to denote the sum of the costs of the edges in $S$.

The $j$ th virtual algorithm associated with the $i$ th multicast is called $\mathrm{VA}_{i, j}$ and is shown in Fig. 1. The goal of $\mathrm{VA}_{i, j}$ is to build a tree $T_{i, j}$ which spans the source $s_{i}$ and some subset of the nodes that requested to join the $i$ th multicast group.

5 This requirement corresponds to a similar requirement in [4]. 
$\mathrm{VA}_{i, j}$

A Initialization ( $s_{i}$ is the root of the $i$ th multicast):

$1 T_{i, j} \leftarrow\left\{s_{i}\right\}$.

2 for all $u$

$\operatorname{PROFIT}_{i, j}(u) \leftarrow 0$;

USED-PROFIT $_{i, j}(u) \leftarrow 0$ (USED-PROFIT $i, j$ is used only for the analysis, specifically in Lemma 5.1).

B Invoked due to receiving profit $\pi$ at node $v$ from $\mathrm{VA}_{i, j-1}\left(\mathrm{VA}_{i, 1}\right.$ is invoked with unit profit due to a join request at node $v$ ):

$1 \operatorname{PROFIT}_{i, j}(v) \leftarrow \operatorname{PROFIT}_{i, j}(v)+\pi$.

2 Contract $T_{i, j}$ to a single vertex $s$.

3 Find smallest $\pi^{\prime} \leqslant \operatorname{PrOFIT}_{i, j}(v)$ such that $\exists$ tree $S$ with the following properties:

(i) $v \in S, s \in S$;

(ii) $\operatorname{Cost}(S) \leqslant\left(\sum_{u \in S, u \neq v} \operatorname{PrOFIT}_{i, j}(u)+\pi^{\prime}\right) / d$.

4 if no such $\pi^{\prime}$ found, then uncontract $T_{i, j}$ and exit

else

4.1 Let $S$ be the tree which satisfies the conditions in Step 3 .

4.2 for all $w \in S, w \neq v$

$\operatorname{USED} \operatorname{PROFIT}_{i, j}(w) \leftarrow \operatorname{PROFIT}_{i, j}(w) ;$

$\operatorname{PROFIT}_{i, j}(w) \leftarrow 0$.

4.3 USED-PROFIT $i, j(v) \leftarrow \pi^{\prime}$;

$\pi \leftarrow \operatorname{PROFIT}_{i, j}(v)-\pi^{\prime} ;$

$\operatorname{PROFIT}_{i, j}(v) \leftarrow 0$.

4.4 Uncontract $T_{i, j}$; $T_{i, j} \leftarrow T_{i, j} \cup S$.

4.5 Update the cost of each edge $e \in S$.

4.6 if $j<L$

Invoke $\mathrm{VA}_{i, j+1}$ by passing a profit of $\pi$ at node $v$ to $\mathrm{VA}_{i, j+1}$.

Fig. 1. The $j$ th virtual phase of the $i$ th real algorithm. Recall that $d$ is the density value defined at the beginning of Section 3.

Each request to join the $i$ th multicast group is considered as a potential unit of profit, and the virtual algorithms use ("consume") this profit to "pay" for their trees. $\mathrm{VA}_{i, j}$ can expand its tree $T_{i, j}$ by adding a subtree only if it can pay for this subtree. We will refer to these subtrees as "fragments". As payment, $\mathrm{VA}_{i, j}$ can use only the profit that is on the nodes of this subtree and that was not used by $\mathrm{VA}_{i, k}$ for $k<j$ (this is denoted by $\operatorname{PROFIT}_{i, j}(v)$ in Fig. 1). More precisely, $\mathrm{VA}_{i, j}$ monitors the profit passed from $\mathrm{VA}_{i, j-1}$. Each time it gets $\pi$ units of profit at some node $v$, it adds $\pi$ to $\operatorname{PROFIT}_{i, j}(v)$. It then tries to find a fragment that includes $v$ such that the ratio of the unused profit associated with nodes of this fragment plus $\pi^{\prime}$ is at least $d=d^{T} \cdot \frac{1}{6 L \log n}$ times the cost of adding this fragment to $T_{i, j}$, where $d^{T}=1 /(m u)$ and $\pi^{\prime} \leqslant \operatorname{PROFIT}_{i, j}(v)$. The goal of the algorithm is to minimize $\pi^{\prime}$.

This subtree is added to the $T_{i, j}, d$ times the cost of this tree is "consumed", and the rest of the profit (in fact, at most one unit) on the newly added nodes is bequeathed to $V_{i, j+1}$.

Observe that, since costs are increasing, the total profit used to construct $T_{i, j}$ is bounded by its final cost divided by $d$. Since $\mathrm{VA}_{i, j}$ builds its tree in an online fashion, there might be a larger (in terms of the spanned nodes that requested participation in $i$ th multicast) tree that can be constructed offline using the same profit. In Lemma 5.1 we show that this 


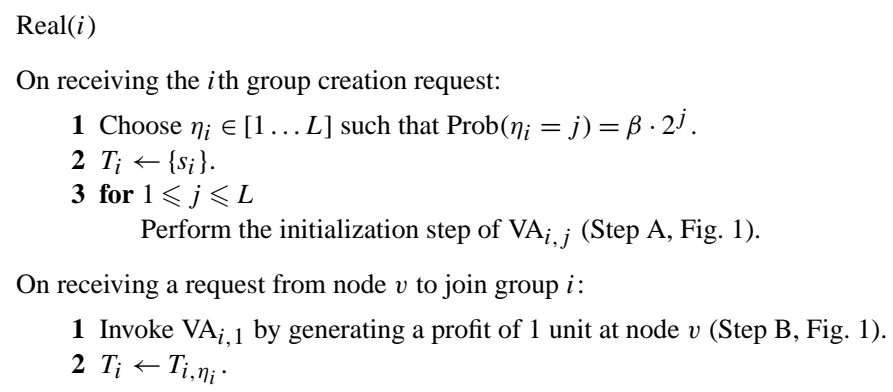

Fig. 2. The real algorithm for multicast group $i$.

"loss" is not very significant. Also, note that the only way virtual algorithms dealing with different multicast groups interact with each other is through edge-costs. Another important property is that for all $j$, the vertices which contribute towards the profit collected by $\mathrm{VA}_{i, j}$ are a subset of the vertices that contribute towards the profit collected by $\mathrm{VA}_{i, j-1}$.

The "real" algorithm is shown in Fig. 2. For each multicast group, it randomly chooses one of the virtual algorithms when the group is created. It then implements the construction of the tree built by this virtual algorithm. We set the probability of choosing $\mathrm{VA}_{i, j}$ to $p_{j}=\beta \cdot 2^{j}$, where $\beta$ is chosen such that $\sum_{j=1}^{L} p_{j}=1$. When a join request is received, the real algorithm invokes $\mathrm{VA}_{i, 1}$ with one unit of profit. If $\mathrm{VA}_{i, 1}$ adds $v$ to its tree, any profit from $v$ that was not consumed in augmenting $T_{i, 1}$ gets passed to $\mathrm{VA}_{i, 2}$, and so on. In this fashion, even though the real algorithm only invokes $\mathrm{VA}_{i, 1}$ explicitly, the algorithms $\mathrm{VA}_{i, j}$ may still get invoked for larger values of $j$. If the chosen virtual algorithm modifies its tree due to this join request, then the real algorithm makes exactly the same modifications to $T_{i}$.

In our analysis, if the $i$ th real algorithm has chosen $\mathrm{VA}_{i, j}$ for a specific multicast, then we do not get credit for the profit that was "used up" when $\mathrm{VA}_{i, j}$ was constructing its tree. Instead, we will only take credit for the profit that was inherited by $\mathrm{VA}_{i, j+1}$.

\section{Edge-costs}

Our algorithm can be viewed as a generalization of the algorithm of Awerbuch and Singh [5]. The main conceptual difference lies in definition of edge-costs. In this section we define the cost metric and the way it is updated as a result of each new request. The cost metric is updated by the virtual algorithms and hence is deterministic.

The online algorithm constructs the cost metric as it goes along. When profit propagates from $\mathrm{VA}_{i, j-1}$ to $\mathrm{VA}_{i, j}$, we consider this an "event". An event might cause $\mathrm{VA}_{i, j}$ to consume some profit and update its tree $T_{i, j}$. Let $c_{e}(k)$ denote the cost of edge $e$ after the $k$ th event. When the $k$ th event occurs, the virtual algorithms use costs $c_{e}(k-1)$ for making their decision. These decisions are then used to compute $c_{e}(k)$ in a deterministic fashion.

Let $\vec{\eta}=\left(\eta_{1}, \ldots, \eta_{\mathcal{M}}\right)$ represent the indices of the virtual algorithms chosen for the $\mathcal{M}$ multicasts. Also, let $p_{\vec{\eta}}$ represent the probability of making this sequence of choices. Define the load on an edge as $1 / u$ times the number of trees it was used in by the real algorithm, 
and let $\lambda_{e}^{(\vec{\eta})}(k)$ represent the load on edge $e$ after the first $k$ events have occurred, where $\vec{\eta}$ represents the choices made by the real algorithms. Since the random choices of the real algorithms for different multicasts are independent, $p_{\vec{\eta}}=\prod_{i=1}^{\mathcal{M}} p_{\eta_{i}}$.

Let $c_{e}(0)=u$ for each edge $e$. Suppose $\operatorname{costs} c(0)$ to $c(k-1)$ were already computed. Then $c_{e}(k)$ is computed as follows.

$$
c_{e}(k)=u \sum_{\vec{\eta}} p_{\vec{\eta}} \mu^{\lambda_{e}^{(\vec{\eta})}(k)} .
$$

The value of $\mu$ is set to $4 m^{6} \log ^{2} \mathcal{M}$. The reason for this value will become clear in Section 6. Observe that, given $\vec{\eta}$, the expression $\lambda_{e}^{(\vec{\eta})}(k)$ is deterministic, and hence the $\operatorname{costs} c_{e}(k)$ are deterministic as well.

Define $X_{e}^{(i, j)}(k)$ as indicator variables, with $X_{e}^{i, j}(k)$ being 1 if edge $e$ is used by $\mathrm{VA}_{i, j}$ during the first $k$ events and 0 otherwise. Notice that $X_{e}^{(i, j)}(k)$ are deterministic quantities. Now, $\lambda_{e}^{(\vec{\eta})}(k)=(1 / u) \cdot \sum_{i=1}^{\mathcal{M}} X_{e}^{\left(i, \eta_{i}\right)}(k)$. We can use this to rewrite the cost $c_{e}(k)$ :

$$
c_{e}(k)=u \sum_{\vec{\eta}} \prod_{i=1}^{\mathcal{M}}\left(p_{\eta_{i}} \cdot \mu^{X_{e}^{\left(i, \eta_{i}\right)}(k) / u}\right) .
$$

Let $\vec{\beta}$ denote the vector $\left\langle\eta_{2}, \eta_{2}, \ldots, \eta_{\mathcal{M}}\right\rangle$. The above sum can now be written as

$$
c_{e}(k)=u\left(\sum_{\eta_{1}=1}^{L} p_{\eta_{1}} \mu^{X_{e}^{\left(i, \eta_{1}\right)}(k) / u}\right) \sum_{\vec{\beta}} \prod_{i=1}^{\mathcal{M}-1}\left(p_{\beta_{i}} \cdot \mu^{X_{e}^{\left(i+1, \beta_{i}\right)}(k) / u}\right) .
$$

Repeating the above step inductively on $\vec{\beta}$, we get

$$
c_{e}(k)=u \prod_{i=1}^{\mathcal{M}} \sum_{j=1}^{L} p_{j} \mu^{X_{e}^{(i, j)}(k) / u} .
$$

The above representation gives an easy way to compute $c_{e}(k)$ efficiently. Since only one of the sums changes during any event, the online algorithm can recompute that sum and obtain the new costs.

The following claim follows from the way we construct the cost metric.

Claim 4.1. The cost $c_{e}(k)$ is the expectation of the quantity $u \mu^{\lambda_{e}(k)}$ where $\lambda_{e}(k)$ is a random variable representing the load on edge e after $k$ events.

\section{Proof of competitiveness}

In order to prove the competitive ratio, we will divide the multicast groups into "profitable" and "unprofitable", based on the cost of the optimum trees for these groups with respect to the cost metric constructed by our algorithm. Here, by optimum trees we mean the trees constructed by the optimum offline algorithm. 
Consider the $i$ th multicast group, and let the number of requests satisfied by the optimal offline algorithm be $r^{*}(i)$. Similarly, let $r(i)$ be the profit obtained by the online algorithm. Let $w^{*}(i)$ be the cost (in the final cost metric) of the tree $T_{i}^{*}$ used by the optimum algorithm to service multicast group $i$. We call a multicast group profitable if the optimal's tree for this multicast group has a high profit to cost ratio in the final cost metric:

Definition 1. The $i$ th multicast group is profitable if $\frac{r^{*}(i)}{w^{*}(i)} \geqslant d^{T}$, where $d^{T}=\frac{1}{m u}$.

We use the quantities $R^{*}$ and $R$ to represent $\sum_{i=1}^{\mathcal{M}} r^{*}(i)$ and $\sum_{i=1}^{\mathcal{M}} r(i)$, respectively. Let $P$ and $U$ represent the set of profitable and unprofitable multicast groups, respectively. Also, we define $R_{P}^{*}=\sum_{i \in P} r^{*}(i)$ and $R_{U}^{*}=\sum_{i \in U} r^{*}(i)=R^{*}-R_{P}^{*}$.

We first show (Lemma 5.2) that the online algorithm obtains almost as much profit from profitable groups as the optimal solution does. Then we show that the total profit obtained by the online algorithm can only be polylogarithmically smaller than optimal's profit from unprofitable groups. To prove the latter claim, we take an indirect route. We use capacity constraints to argue that the quantity $m R_{U}^{*}$ is bounded by the sum of the final costs of all edges (Lemma 5.3). Finally, we bound the final costs in terms of the expected profit obtained by the online algorithm (Lemma 5.7).

Consider the quantities $\operatorname{ProfIT}_{i, j}(v)$ and $\operatorname{USED}_{-\operatorname{PrOFIT}_{i, j}}(v)$ at the end, i.e., after all requests have been received. Let $\mathrm{P}_{i, j}(v)=\operatorname{PROFIT}_{i, j}(v)+\operatorname{USED}_{\operatorname{PROFIT}}, \mathrm{PR}_{i}(v)$. Informally, the quantity $\mathrm{P}_{i, j}(v)$ denotes the profit consumed by $\mathrm{VA}_{i, j}$ at node $v$. For any set $X$ of vertices, $\mathrm{P}_{i, j}(X)=\sum_{v \in X} \mathrm{P}_{i, j}(v)$; the definitions of PROFIT $i, j$ and UsEd-PROFIT $i, j$ are similarly extended.

The following lemma was first proved by [5] with worse constant factors. Our proof is simpler, and allows us to show (Appendix A) that the algorithm can be implemented in polynomial time.

Lemma 5.1. $\mathrm{P}_{i, j}\left(T_{i}^{*}\right) \leqslant 3 w^{*}(i) d \log n$.

Proof. We first bound the quantity $\operatorname{PROFIT}_{i, j}\left(T_{i}^{*}\right)$. This contribution comes from nodes in $T_{i}^{*}$ which do not belong to $T_{i, j}$. The profit consumed on these nodes by $\mathrm{VA}_{i, j}$ must be at most $w^{*}(i) d$, else these nodes would have formed a fragment on their own and been added to $T_{i, j}$.

Now we bound USED-PROFIT $i, j\left(T_{i}^{*}\right)$. This contribution comes from nodes that belong to $T_{i, j}$. Recall that $\mathrm{VA}_{i, j}$ acquires $T_{i, j}$ in tree fragments. Consider an Eulerian tour $D$ of $T^{*}$. Let a segment of tour $D$ be a maximal contiguous piece of $D$ such that all edges of the segment belong to the same fragment of $T_{i, j}$. Initially, all segments are marked active. If two consecutive active segments on this tour belong to the same fragment, they are merged together along with the portion of the tour between them to form a single segment. Let $t(s)$ denote the event at which the edges of segment $s$ were added to $T_{i, j}$.

Furthermore, we define a pred and succ relation on active segments such that $\operatorname{pred}(s, D)$ is the predecessor of $s$ in tour $D$ and $\operatorname{succ}(s, D)$ is the successor of $s$ in $D$.

Let $D_{0}=D$. For $h \geqslant 1$, let $\mathcal{H}_{h}=\left\{s\right.$ is an active segment of $D_{h-1}, t(s)<t(\operatorname{pred}(s$, $\left.\left.\left.D_{h-1}\right)\right), t(s)<t\left(\operatorname{succ}\left(s, D_{h-1}\right)\right)\right\}$. Let $\mathcal{L}_{h}$ denote the remaining segments of $D_{h-1}$, and let 
$D_{h}$ denote the tour $D_{h-1}$ with each segment in $\mathcal{L}_{h}$ marked inactive. The segments in $\mathcal{H}_{h}$ remain active in $D_{h}$. As mentioned above, consecutive active segments are merged if they belong to the same fragment.

Note that for all $h$ :

$$
\left|\mathcal{L}_{h}\right|>\left|\mathcal{H}_{h}\right| \text {. }
$$

This implies that there at $\operatorname{most} \log n$ non empty sets $\mathcal{L}_{h}$. Let $s \in \mathcal{L}_{h}$ for some $h$. Also, let $s^{\prime}$ be the successor or predecessor segment of $s$ in $D_{h-1}$ with $t\left(s^{\prime}\right)<t(s)$ and let $p$ consist of the part of $D$ between $s$ and $s^{\prime}$.

Assume USED-PROFIT $i, j(s)>d\left(w^{*}(s)+w^{*}(p)\right)$. Let $v \in s$ be the node with the last request in multicast $i$ among all nodes in $s$. When the request at $v$ arrived, the sum $\operatorname{PROFIT}_{i, j}(s)=\sum_{u \in s} \operatorname{PROFIT}_{i, j}(u)$ is more than $d\left(w^{*}(s)+w^{*}(p)\right)$, because $\operatorname{PROFIT}_{i, j}(s)$ is the source of USED-PROFIT $i, j(s)$. Thus, at that time we could have used at most $d\left(w^{*}(s)+w^{*}(p)\right)$ to add $s+p$ as a fragment. Since the algorithm always tries to create a fragment using the minimum amount of profit, we have:

$$
\operatorname{USED}_{\operatorname{PROFIT}}, j(s) \leqslant d\left(w^{*}(s)+w^{*}(p)\right) .
$$

Considering that $D$ visits every node twice it follows that

$$
\sum_{s \in \mathcal{L}_{h}} \operatorname{USED}^{-P_{R O F I T}, j}(s) \leqslant 2 w^{*}(i) d .
$$

Summing over all values of $h$ it follows that the profit consumed by $\mathrm{VA}_{i, j}$ from all nodes which belong to $T^{*} \cap T_{i, j}$ is at most $2 w^{*}(i) d \log n$. This completes the proof of this lemma.

We now show that for profitable multicasts, the profit obtained by our online algorithm is high.

Lemma 5.2. $R \geqslant R_{P}^{*} / 2$.

Proof. Since there are $L$ levels, Lemma 5.1 guarantees that the total wasted profit for multicast group $i$ is at most $3 L w^{*}(i) d \log n$. Plugging in $d=d^{T} \cdot \frac{1}{6 L \log n}$ and using the fact that $i$ profitable implies that $r^{*}(i) \geqslant d^{T} w^{*}(i)$, we obtain a bound of $r^{*}(i) / 2$ on the wasted profit. Therefore, $r(i) \geqslant r^{*}(i) / 2$ for all profitable groups $i$. Summing over all the profitable groups, we get the desired result.

Having bounded the profit from the profitable groups, we now concentrate on the unprofitable groups. Recall that $c_{e}$ is the cost of edge $e$ at the end, i.e., after all the events have taken place, and that the costs are non-decreasing in time.

Lemma 5.3. $m R_{U}^{*} \leqslant \sum_{e} c_{e}$.

Proof. Let $k_{e}^{*}$ be the number of multicast groups which use edge $e$ in the optimal offline solution. Consider the tree $T_{i}^{*}$ used by the optimal solution to route the $i$ th multicast group. 
If this group is unprofitable then by definition $r^{*}(i) \leqslant \frac{1}{m u} \sum_{e \in T_{i}^{*}} c_{e}$. We sum this over all the unprofitable multicast groups, and then reverse the order of summation.

$$
R_{U}^{*} \leqslant \frac{1}{m u} \sum_{i \in U} \sum_{e \in T_{i}^{*}} c_{e} \leqslant \frac{1}{m u} \sum_{e} k_{e}^{*} c_{e} \leqslant \frac{1}{m} \sum_{e} c_{e} .
$$

The last inequality follows from the fact that the optimal offline is not allowed to exceed capacities, implying $k_{e}^{*} \leqslant u$.

Let $w_{j}(i)$ represent the cost incurred by $\mathrm{VA}_{i, j}$ in constructing the tree $T_{i, j}$. In other words, each tree fragment of $T_{i, j}$ contributes to $w_{j}(i)$ its cost associated with the event of adding this fragment. We use $w(i)$ to denote $w_{\eta(i)}(i)$, where $\eta$ represents the choice of the real algorithm. Let $r_{j}(i)$ represent the profit consumed in constructing this $T_{i, j}$. The following lemma implies that if the expected profit is small, then the expected cost of the constructed trees is small as well.

Lemma 5.4. $\mathbf{E}(r(i)) \geqslant(d / 2) \mathbf{E}(w(i))-\frac{1}{\mathcal{M}}$, where $w(i)$ is the cost paid by the real algorithm for multicast group $i$.

Proof. If the real algorithm chooses to follow $\mathrm{VA}_{i, j}$, i.e., $\eta(i)=j$, then it will get at least the profit used by $\mathrm{VA}_{i, j+1}$. Therefore:

$$
\mathbf{E}(r(i)) \geqslant \sum_{j=1}^{L-1} p_{j} r_{j+1}(i) .
$$

By definition, $p_{j}=p_{j+1} / 2$, and hence

$$
\mathbf{E}(r(i)) \geqslant \sum_{j=2}^{L} p_{j} r_{j}(i) / 2 .
$$

By construction:

$$
\mathbf{E}(w(i))=\sum_{j=1}^{L} p_{j} w_{j}(i) \leqslant(1 / d) \sum_{j=1}^{L} p_{j} r_{j}(i) .
$$

Thus, we have

$$
d \cdot \mathbf{E}(w(i)) \leqslant 2 \mathbf{E}(r(i))+p_{1} r_{1}(i) .
$$

Now notice that $r_{1}(i)$ can be at most $n$, since each request brings in one unit of profit, and there can be at most $n$ requests for a single multicast group. Also, $p_{1} \sum_{j=1}^{L} 2^{j-1}=1$, which implies that $p_{1} / 2<2^{-L}$. Substituting $L=\log n+\log \mathcal{M}$, we obtain $\mathbf{E}(r(i)) \geqslant$ $(d / 2) \mathbf{E}(w(i))-\frac{1}{\mathcal{M}}$.

We remark that in this proof the fact that the VAs are deterministic is quite crucial; otherwise, the profits $r_{j}(i)$ would be conditioned on the random choices made by the real algorithms and the above argument would break down completely. 
Now we prove that if the expected cost of the constructed trees is small, then the total cost of all the edges is small as well. But first, we need to prove the following technical lemma. Roughly speaking, this lemma implies that if an event caused an edge to be used by one of the trees, the increase in the cost of this edge is proportional to its current cost.

Consider an event $k$ that caused $\mathrm{VA}_{i, j}$ to augment its tree, and let $E_{k}$ represent the set of edges of the newly added subtree.

Lemma 5.5. For all $e \in E_{k}, c_{e}(k)-c_{e}(k-1) \leqslant \frac{\log \mu}{u} p_{j} c_{e}(k-1)$. For the edges $e \notin E_{k}$, $c_{e}(k)=c_{e}(k-1)$.

Proof. The second part of the lemma is obvious. We concentrate on edges $e \in E_{k}$. By definition of the indicator variables, $X_{e}^{(i, j)}(k-1)=0$ and $X_{e}^{(i, j)}(k)=1$. Using Eq. (1), we have:

$$
\begin{aligned}
c_{e}(k)-c_{e}(k-1) & =p_{j}\left(\mu^{X_{e}^{(i, j)}(k) / u}-1\right) u \prod_{i^{\prime} \neq i} \sum_{j^{\prime}} p_{j^{\prime}} \mu^{X_{e}^{\left(i^{\prime}, j^{\prime}\right)}(k) / u} \\
& =p_{j}\left(\mu^{1 / u}-1\right) \frac{c_{e}(k-1)}{\sum_{j^{\prime}} p_{j^{\prime}} \mu^{X_{e}^{\left(i, j^{\prime}\right)}(k-1) / u}} \\
& \leqslant p_{j}\left(\mu^{1 / u}-1\right) c_{e}(k-1) .
\end{aligned}
$$

The last inequality above follows from the fact that $\sum_{j^{\prime}} p_{j^{\prime}} \mu^{X_{e}^{\left(i, j^{\prime}\right)}(k-1) / u} \geqslant \sum_{j^{\prime}} p_{j^{\prime}}=1$. For all $x$ between 0 and $1,2^{x}-1 \leqslant x$. Therefore, $\mu^{1 / u}-1=2^{(\log \mu) / u}-1 \leqslant(\log \mu) / u$, which completes the proof of the lemma.

Let $W=\sum_{i} w_{i}$ represent the total cost of the trees constructed by the online algorithm. The following lemma relates the cost incurred by the algorithms and the final cost of the edges.

Lemma 5.6. $\frac{\log \mu}{u} \mathbf{E}(W) \geqslant \sum_{e}\left(c_{e}-u\right)$.

Proof. Let $\Delta_{e}(k)=c_{e}(k)-c_{e}(k-1)$ represent the increase in cost on edge $e$ during the $k$ th event. Clearly, $c_{e}=c_{e}(0)+\sum_{k} \Delta_{e}(k)$ where the summation is over all events and $c_{e}(0)=u$ for all edges $e$. Now, let $\mathrm{VA}_{i_{k}, j_{k}}$ be the virtual algorithm that updates its tree during event $k$. Lemma 5.5 implies that

$$
\sum_{e}\left(c_{e}-u\right) \leqslant(\log \mu / u) \sum_{i} \sum_{j} p_{j} \sum_{k: i_{k}=i, j_{k}=j} \sum_{e \in E_{k}} c_{e}(k-1) .
$$

Using definition of $w_{j}(i)$, we can rewrite this expression as follows:

$$
\sum_{e}\left(c_{e}-u\right) \leqslant(\log \mu / u) \sum_{i} \sum_{j} p_{j} w_{j}(i)=(\log \mu / u) \sum_{i} \mathbf{E}(w(i)) .
$$

Using linearity of expectations, $\sum_{i} \mathbf{E}(w(i))=\mathbf{E}\left(\sum_{i} w_{i}\right)$, which completes the proof. 
We are now ready to show that if the obtained profit is small, then the total cost of all the edges is small as well.

Lemma 5.7. $\left(5 \frac{d^{T}}{d} \log \mu\right) m \mathbf{E}(R) \geqslant \sum_{e} c_{e}$

Proof. Summing up Lemma 5.4 over all multicast groups, we have:

$$
\frac{2}{d}\left(\mathbf{E}(R)+\sum_{i=1}^{\mathcal{M}} \frac{1}{\mathcal{M}}\right) \geqslant \mathbf{E}(W)
$$

As we will show below, $\mathbf{E}(R) \geqslant 1$. Therefore, the above inequality can be rewritten as $\frac{4}{d} \mathbf{E}(R) \geqslant \mathbf{E}(W)$. Using Lemma 5.6, and the fact that $m d_{T} u=1$, we obtain

$$
\sum_{e} c_{e} \leqslant \frac{\log \mu}{u} \cdot \frac{4}{d} \mathbf{E}(R)+m u=m \log \mu\left(\frac{4 d_{T}}{d} \mathbf{E}(R)+\frac{u}{\log \mu}\right) .
$$

To complete the proof, it remains to show that the first $u / \log \mu$ requests are always accepted, i.e., $\mathbf{E}(R) \geqslant u / \log \mu$. Suppose the first $k<u / \log \mu$ requests have been accepted. As a result, the load on each edge is no more than $k$, and the cost of servicing the next request can be at most $m u \mu^{k / u}<m u \mu^{1 / \log \mu}=2 m u$. By construction, the profit needed to pay for this cost is at most

$$
2 m u d=2 m u \frac{1}{m u} \frac{1}{6 L \log n}=\frac{1}{3 L \log n} .
$$

Thus, the unit of profit brought by this request is enough to pay for extending the trees of all VA algorithms dealing with the corresponding multicast group. Thus, this request is going to be accepted by the real algorithm as well. In other words, if there are less than $u / \log \mu$ requests generated by the adversary then the real algorithm accepts them all and has a competitive ratio of 1 . Else, $R$ (and therefore $\mathbf{E}(R)$ ) is greater than $u / \log \mu$, which completes the proof of the claim.

Combining Lemmas 5.3 and 5.7 with Lemma 5.2, we obtain the following result:

Theorem 5.1. If $R^{*}$ is the number of multicast join requests accepted by the optimum offline algorithm and $R$ is the number of multicast join requests accepted by our online algorithm, then $R^{*} / \mathbf{E}(R)=O(\log n \log \mu(\log n+\log \mathcal{M}))$.

Appendix A explains how to implement each decision step of our online algorithm in polynomial time.

\section{Capacity constraints}

In the previous section we showed that the algorithm accepts a significant fraction of the requests accepted by the optimum offline algorithm. It remains to show that our online algorithm does not overflow the available capacities. To that end, we set $\mu=4 m^{6} \log ^{2} \mathcal{M}$. 
Note that, by Theorem 5.1, this implies that we get an $O(\log n(\log n+\log \log \mathcal{M})(\log n+$ $\log \mathcal{M})$ )-competitive algorithm. For the special case where each node is allowed to serve at most one multicast group, we clearly have an $O\left(\log ^{3} n\right)$-competitive algorithm.

We now show that the above value of $\mu$ is sufficient to ensure that the capacity constraints are never violated with high probability.

Lemma 6.1. For any edge $e$, the cost $c_{e}$ does not exceed $u \mu^{1 / 2}$.

Proof. Suppose $c_{e}(k)>u \mu^{1 / 2-1 / u}$ for some $k$. Since $\mu=4 m^{6} \log ^{2} \mathcal{M}$ and $u \geqslant \log \mu$, we get $c_{e}>u m^{3} \log \mathcal{M}$. Since maximum profit of a single tree fragment is $n$, this cost is above maximum profit divided by $d$. Thus, this edge will never be used again by any VA. The claim follows from the fact that during any one event, $c_{e}(k)$ can increase only by a factor of $\mu^{1 / u}$.

Lemma 6.2. With probability at least $1-1 / \mathrm{m}^{2}$, no edge violates its capacity constraint.

Proof. Claim 4.1 states that $c_{e}$ is equal to the expected value of the quantity $u \mu^{\lambda_{e}}$, where $\lambda_{e}$ is the final load on an edge. The event $\lambda_{e} \geqslant 1$ implies that $u \mu^{\lambda_{e}} \geqslant \mu^{1 / 2} \mathbf{E}\left(u \mu^{\lambda_{e}}\right)$. Using Markov inequality, the probability of this event happening is at most $\mu^{-1 / 2}<1 / \mathrm{m}^{3}$. Therefore, with probability at least $1-1 / \mathrm{m}^{2}$, all edges satisfy the capacity constraints.

If the algorithm tries to exceed capacity of an edge, we terminate it. Lemma 6.2 guarantees that this does not affect the asymptotic competitive ratio given in Theorem 5.1.

\subsection{Relaxing some of the assumptions}

Some of the assumptions made in Section 2 can be relaxed using techniques from [4]. In particular, we can handle the case where the bandwidth requirements of different multicast groups, the capacities of different edges, and the profits of different multicast groups can vary arbitrarily. The modifications to our algorithm, analysis, and competitive ratio proceed similarly to those in [4].

\section{Lower bounds}

The competitive ratio of our algorithm holds against a semi-oblivious adversary-the adversary is allowed to look at the multicast tree generated by the online algorithm but only after all the requests for that multicast group have been processed. A semi-oblivious adversary is at least as powerful as an oblivious one, so our competitive ratio holds against an oblivious adversary as well. For the purpose of proving lower bounds it is desirable to use the less powerful adversary; accordingly we first show a lower bound of $\Omega(\log \mathcal{M} \log n)$ against an oblivious adversary. Then we show that a polynomial lower bound on the competitive ratio of any online algorithm against an adaptive online adversary. 


\subsection{Against an oblivious adversary}

A lower bound of $\log n$ for the problem studied in this paper immediately follows from [4]. The challenge in the online multicast problem is to decide which requests to service ("winner picking") and how to route a request ("online routing"). We now show how to combine a lower bound for winner picking [3] with the lower bound for online routing [4] to achieve a lower bound of $\log \mathcal{M} \log n$ for the online multicast problem. This is the first lower bound stronger than $\log n$ for the online multicast problem if the bandwidth requested by each multicast can be significantly smaller than bandwidth of a single link.

Theorem 7.1. No algorithm for selective online multicast can have a competitive ratio better than $\Omega(\log (\mathcal{M} / u) \log n)$ even against an oblivious adversary, and even when the requests are non-interleaved.

Proof. The basic idea behind the winner-picking lower bound for online multicast is the following. Assume $\mathcal{M}$ multicasts are created, but both the online and the offline algorithm are just allowed to pick one. A multicast consists of at least one and up to $\log \mathcal{M}$ classes, each class consisting of $c$ requests for some parameter $c$. Half of the multicasts, chosen randomly from all multicasts, consist of exactly $c$ request. One fourth of the multicasts, chosen randomly from the remaining half of the multicasts, consist of exactly $2 c$ request, etc. Thus, the expected profit of online is $2 c$, while the expected profit of offline is $c \log \mathcal{M}$.

The lower bound for online routing works in phases: there are $\log n+1$ phases, with the "profit", i.e., number of requests, doubling in each phase. It can be shown that there must be a phase such that the expected profit that online has received so far is at most $2 / \log n$ of the profit that is available in the current phase. In this phase, offline services all the request, i.e., takes all the profit, and the sequence of requests terminates.

We show next how to combine these two bounds. To simplify the presentation we assume that all demands and all edge capacities are 1 , but it is permissible to satisfy a fractional demand and obtain a fractional profit (the profit for a multicast group is the product of the satisfied demand and the number of satisfied requests). We explain later how this result carries over to our model.

We restrict ourselves to values of $\mathcal{M}$ such that $\sqrt{n}>\log \mathcal{M}$. Consider the graph $G$ on $n+2$ vertices (see Fig. 3) which is defined as follows. The vertex set is $\left\{r, x, v_{1}, \ldots, v_{n}\right\}$. There is an edge from $r$ to $x$, and there is an edge from $x$ to each of $v_{1} \ldots v_{n}$. For convenience, define $M=\mathcal{M} / \log n$ and $N=n / \log M$. Notice that the restriction we have placed on $\mathcal{M}$ implies that $N>\sqrt{n}$.

The adversary operates in at most $\log N$ phases: we describe the $i$ th phase, $1 \leqslant i \leqslant$ $\log N$. In phase $i$ the adversary divides the vertices $v_{1} \ldots v_{n}$ into classes of size $2^{i-1}$. Notice that there must be at least $\log M$ classes. The adversary then generates $M$ multicasts, each with $r$ as the root. The requests for these multicasts will be non-interleaved. For each multicast, the adversary generates a request at each of the nodes in the first class. Then the adversary flips a coin. If the coin toss is a Head (i.e., with probability half) the adversary moves on to the next multicast. Else, it generates a request at each node in the next class, flips another coin, and repeats the same process again. If requests have been generated at $\log M$ classes for the same multicast, the adversary moves on to the next multicast. At the 


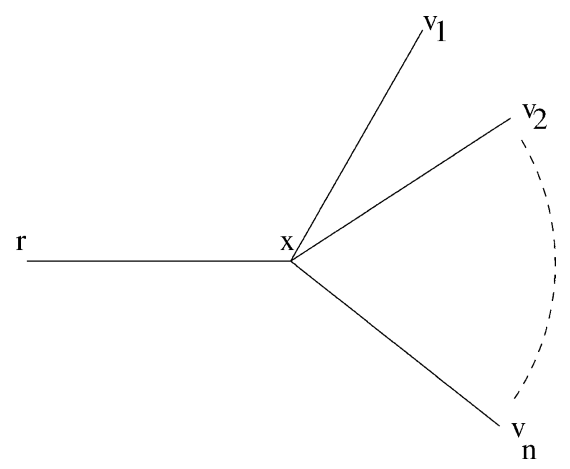

Fig. 3. The lower bound graph for Theorems 7.1 and 7.4.

end of all $M$ multicasts for this phase, the adversary moves on to the next phase. Notice that setting the class size to $2^{i-1}$ is equivalent to doubling available profit by 2 for each phase.

Let $c(i)$ be the capacity on the edge $(r, x)$ used by the online algorithm during phase $i$. Also, let $p(i)$ be the profit obtained by online during the $i$ th phase. Let $p^{*}(i)$ and $c^{*}(i)$ be the corresponding quantities for the solution generated by the oblivious adversary. Notice that $\sum_{i} c(i)$ can be at most 1 . Define $S(k)=\frac{1}{2^{k}} \sum_{1 \leqslant i \leqslant k} \mathbf{E}(p(i))$. The total expected profit obtained by the algorithm in the first $k$ phases is $2^{k} S(k)$.

The following two claims now hold:

Claim 7.2. $\mathbf{E}(p(i))<2^{i} \mathbf{E}(c(i))$.

Proof. Suppose the online algorithm decides to satisfy a fractional demand of $x$ for a specific multicast in the $i$ th phase. The cost incurred is $x$. Suppose that this commitment is made by the algorithm after the $j$ th request for this multicast group comes in. Then the expected profit from this multicast group is $2^{i-1} \cdot x \sum_{j \leqslant j^{\prime} \leqslant M} 2^{j^{\prime}-j}<2^{i} x$. Now we sum this up over all the multicast groups in phase $i$ to get the desired result.

Claim 7.3. During any phase $i$, the adversary can ensure that $\mathbf{E}\left(p^{*}(i)\right) \geqslant \frac{\log M}{4} 2^{i} c^{*}(i)$.

Proof. During phase $i$, the adversary can pick the multicast with the maximum number of classes of requests. Let $P^{<}(i)$ denote the probability of this number being less than $i$. Now, $P^{<}(i)=\left(1 / 2+1 / 4+\cdots+2^{1-i}\right)^{M}=\left(1-2^{1-i}\right)^{M}$, for $i<\log M$. Clearly, $P^{<}\left(\frac{2}{3} \log M\right)<$ $1 / 3{ }^{6}$ This tells us that the expected number of classes is greater than $\frac{\log M}{2}$. To complete the proof of this claim we observe that each class in the $i$ th phase has $2^{i-1}$ requests.

We now prove that there exists a phase $k$ such that the total expected profit obtained by the online algorithm during the first $k$ phases is no more than $2^{k+1} / \log N$. Suppose this is

$\overline{6} P^{<}\left(\frac{2}{3} \log M\right)$ is in fact much smaller, but we do not need a stronger bound. 
not true. Then, $S(i)>2 / \log N$ for all $i$. In particular, $\sum_{1 \leqslant i \leqslant \log N} S(i)>2$. But $\sum_{i} S(i)=$ $\sum_{i} \mathbf{E}(p(i)) \sum_{i \leqslant j \leqslant \log N} \frac{1}{2^{j}} \leqslant 2 \sum_{i} \mathbf{E}(p(i)) / 2^{i}$. Using Claim 7.2, we have $\sum_{i} \mathbf{E}(c(i))>1$. But this is a contradiction, as the online is not allowed to overflow capacities. This proves the existence of a phase $k$ with $S(k) \leqslant 2 / \log N$.

The oblivious adversary cannot see the coin tosses of the online algorithm but it can compute in advance the quantities $S(i)$. Having found the value $k$ guaranteed by the above argument, the adversary stops after phase $k$ and does not generate any more multicast requests. The adversary also generates a "good" solution as follows: it does not satisfy any demands in the first $k-1$ phases, and in the last phase, it uses up the entire edge $(r, x)$. Now from Claim 7.3, $\mathbf{E}\left(p^{*}(k)\right) \geqslant 2^{k-2} \log M$. The total expected profit obtained by the online algorithm is $2^{k} S(k) \leqslant 2^{k+1} / \log N$. This gives a lower bound of $\Omega(\log M \log N)$ on the competitive ratio of any online algorithm. Since $N \geqslant \sqrt{n}$ and $M=\mathcal{M} / \log n$, this is also a $\Omega(\log \mathcal{M} \log n)$ lower bound.

In the above analysis, we assumed that $\sqrt{n}>\log \mathcal{M}$. This is not a very restrictive assumption, because for $\log \mathcal{M}>\sqrt{n}$, our proof shows that the competitive ratio is already as bad as $\Omega(\sqrt{n})$.

Now we adapt this lower bound proof to our model. Assume that the capacity is $u$. Let $\mathcal{M}$ be the number of multicasts, and let $\mathcal{M}^{\prime}=\mathcal{M} / u$. The adversary proceeds as before, except that each phase gets repeated $u$ times. Also, the online algorithm is restricted to satisfy the entire demand of 1 unit or none at all. The same calculation as done above gives a lower bound of $\Omega\left(\log \mathcal{M}^{\prime} \log n\right)=\Omega(\log (\mathcal{M} / u) \log n)$.

\subsection{Against an adaptive-online adversary}

The next obvious question to ask is whether any algorithm can work well against a more powerful adversary. We answer this question in the negative in this section. Recall that an adaptive-online adversary is one which can adapt the input sequence depending on the response of the online algorithm; however the adversary must also generate a solution as it goes along.

Theorem 7.4. No randomized algorithm for selective online multicast can have better than $\Omega\left(\min \left(n, \frac{\mathcal{M}}{u}\right)\right)$ competitive ratio against an adaptive-online adversary. The lower bound holds even when the requests are non-interleaved.

Proof. Consider the same graph as in the previous section (see Fig. 3). Each edge has a capacity $u$, all requests have demand 1 . The value of $\mathcal{M}$ is fixed at $2 n u$. The adversary works in $2 u$ phases.

During the $i$ th phase, the adversary chooses a number $m_{i}$ uniformly at random from the set $\{1, \ldots, n\}$. The adversary then does the following for at most $m_{i}-1$ sub-phases. It generates a new set-up request at $r$. It then generates, in sequence, requests at nodes $v_{1}, \ldots, v_{n}$ for the newly set up multicast group. As soon as the online algorithm accepts any of these requests, the adversary aborts this phase completely and moves to the next phase. If the online algorithm does not accept any of these $n$ requests, the adversary moves to the next sub-phase. The adversary does not accept any request during the first $m_{i}-1$ sub-phases. If all $m_{i}-1$ sub-phases end without the online algorithm having accepted 
even one request, then the adversary generates yet another set-up request at node $r$. It then generates requests in sequence at nodes $v_{1}, \ldots, v_{n}$ and accepts them all without bothering about the online algorithm (if the edge $\langle r, x\rangle$ is already full in the adversary's network, the adversary rejects these requests). During any phase, the online algorithm gets a profit of $n$ if it "guesses" correctly the value $m_{i}$, and at most 1 otherwise. Since $m_{i}$ is chosen uniformly at random from $\{1, \ldots, n\}$, the expected profit for the online algorithm during any one phase is at most 2 .

At the end of these $2 u$ phases, the adversary is guaranteed to have accepted $u n$ requests. The expected number of requests accepted by the online algorithm can be no more than $4 u$. This proves the desired result. Notice that the requests generated by the adversary are noninterleaved. If each node is allowed to serve no more than one multicast group, the above argument can be modified to obtain a $\Omega(\sqrt{n})$ lower bound for $u=O(\sqrt{n})$.

\section{Appendix A. Making our algorithm run in polynomial time}

There are two issues we need to address to make our algorithm run in polynomial time. First, in Step 3 of $\mathrm{VA}_{i, j}$ (Fig. 1), we need to compute the minimum profit needed out of the new request to create an appropriate tree fragment. Second, we need to provide a polynomial time approximation algorithm for finding the fragment in Step 3 of $\mathrm{VA}_{i, j}$.

The first problem can be solved by doing a binary search. Let $\pi$ be the available profit on the node where the new request has been generated. We run a maximal dense tree algorithm with all $\pi$ units of profit available. If a non-empty tree is returned, we try with half the profit, and so on. The key observation here is that we can afford to incur an additive error of $1 /(8 n L)$ in finding the minimum profit $\pi^{\prime}$. Therefore, the binary search needs to be done only up to a depth $\log 8 \pi n L$. The competitive ratio remains unaffected by this modification.

The second problem is resolved using the prize-collecting Steiner tree algorithm of Goemans and Williamson [13] which has some very nice properties [11,12].The prize collecting Steiner tree problem is the following: given a cost metric on the edges, and a penalty function defined on the vertices, find a tree such that the sum of the cost of the edges in the tree and the penalty of the vertices not in the tree is minimum. We already have a cost metric defined on edges. For a vertex $v$ with available profit $\pi_{v}$, the penalty of missing $v$ is set to $\pi_{v} / d$. Now the approximation algorithm [13] for the prize-collecting Steiner tree problem is invoked, and the tree fragment returned by this algorithm is used. If $d$ is set to half its current value, we obtain the same bound on the competitive ratio (except the loss of a factor of two).

\section{A.1. The prize-collecting Steiner tree problem}

The problem is the following: given a graph $G(V, E)$ with a cost function $c$ defined on the edges, a set of destinations $D$, and a prize function $\pi$ defined on $D$, find a tree $T$ that minimizes

$$
\sum_{e \in T} c(e)+\sum_{v \in D, v \notin T} \pi(v) .
$$


We use $C_{T}$ to denote the first component of the cost and $P_{T}$ for the second component. This problem is NP-hard, being a strict generalization of the Steiner tree problem. A 2-approximation for this problem was given by Goemans and Williamson [13]. Their approximation generates trees $T$ which have the following nice property:

$$
C_{T}+2 P_{T} \leqslant 2\left(C_{T^{*}}+P_{T^{*}}\right)
$$

where $T^{*}$ is the optimal tree. This property was first used by Goemans and Kleinberg [12] and a proof of this property is presented in [11]. This property guarantees, as we will see, that with appropriately defined prizes, the prize-collecting Steiner tree approximation given by [13] is also a good enough approximation to the maximal dense subtree problem.

\section{A.2. Using the prize-collecting Steiner tree problem to make our algorithm polynomial time}

Suppose the prize-collecting algorithm is run with $\pi(u)=\operatorname{PROFIT}_{i, j}(u) / d$, returning a tree $T$ with cost $w$ and profit $r$, where the quantities $\operatorname{PROFIT}_{i, j}(u)$ are as defined in Step 3 of Fig. 1. Property A.1 gives the following two results.

Claim A.1. $w \leqslant 2 r / d$.

Claim A.2. Let $T^{\prime}$ be any tree, with cost $w^{\prime}$ and profit $r^{\prime}$. Then $\left(r^{\prime}-r\right) / d \leqslant w^{\prime}$.

In particular, Claim A.2 implies that if $T$ is empty then for any tree $T^{\prime}, w^{\prime} \geqslant r^{\prime} / d$. Now we make the following changes to the algorithms $\mathrm{VA}_{i, j}$ defined in Fig. 1. First, we define $d=d^{T} \cdot \frac{1}{32 L \log n}$, which is half of the previous value. Also, instead of finding the maximal dense subtree, $\mathrm{VA}_{i, j}$ repeatedly invokes the prize-collecting algorithm, contracts the tree found, and runs the prize-collecting algorithm again, till the prize-collecting algorithm returns an empty tree. Claims A.1 and A.2 are now sufficient to guarantee the competitive ratio in Theorem 5.1.

\section{A.3. The selective batch arrival problem}

Awerbuch, Azar, and Plotkin [4] show how to solve the semi-selective batch arrival problem: all join requests arrive in batches and they must be either all accepted or all rejected. They use a cost metric very similar to the one used by us in this paper. Each time a new request arrives, they find the minimum Steiner tree (within a factor of 2) that can satisfy these requests. If this tree is cheap enough, they accept the multicast and update the costs. We make the following modification: assign to each vertex that wants to be a part of the multicast a prize equal to the profit from that vertex, and then run a single instance of the prize-collecting algorithm. The tree so obtained is used to service the current multicast. Claims A.1 and A.2 are sufficient to give the same (up to constant factors) bound as [4]. 


\section{References}

[1] J. Aspnes, Y. Azar, A. Fiat, S. Plotkin, O. Waarts, On-line load balancing with applications to machine scheduling, in: 25th ACM Symposium on Theory of Computing, 1993, pp. 623-631.

[2] B. Awerbuch, Y. Azar, A. Blum, S. Vempala, New approximation guarantees for minimum weight $k$-trees, SIAM J. Comput. 28 (1) (1998) 254-262.

[3] B. Awerbuch, Y. Azar, A. Fiat, T. Leighton, Making commitments in the face of uncertainty: how to pick a winner almost every time, in: 28th ACM Symposium on Theory of Computing, 1996, pp. 519-530.

[4] B. Awerbuch, Y. Azar, S. Plotkin, Throughput competitive online routing, in: 34th IEEE Symposium on Foundations of Computer Science, 1993, pp. 32-40.

[5] B. Awerbuch, T. Singh, Online algorithms for selective multicast and maximal dense trees, in: 29th ACM Symposium on Theory of Computing, 1997.

[6] Y. Azar, A. Broder, A. Karlin, On-line load balancing, Theoret. Comput. Sci. 130 (1) (1994) 73-84.

[7] Y. Bartal, A. Fiat, S. Leonardi, Lower bounds for on-line graph problems with application to on-line circuit and optical routing, in: Proc. of the 28th Symposium on Theory of Computation, 1996, pp. 531-540.

[8] A. Blum, R. Ravi, S. Vempala, A constant factor approximation for the k-mst problem, J. Comput. System Sci. 58 (1) (1999) 101-108.

[9] J. Garay, I. Gopal, S. Kutten, Y. Mansour, M. Yung, Efficient on-line call control algorithms, J. Algorithms 23 (1) (1997) 180-194.

[10] J. Garay, I. Gopal, Call preemption in communication networks, in: Proc. INFOCOM '92, vol. 44, Florence, Italy, 1992, pp. 1043-1050.

[11] N. Garg, A 3-approximation for the minimum tree spanning $k$ vertices, in: 37th IEEE Symposium on Foundations of Computer Science, 1996, pp. 302-309.

[12] M. Goemans, J. Kleinberg, An improved approximation ratio for the minimum latency problem, in: 7 th ACM-SIAM Symposium on Discrete Algorithms, 1996, pp. 152-158.

[13] M. Goemans, D. Williamson, A general approximation technique for constrained forest problems, SIAM J. Comput. 24 (2) (1995) 296-317.

[14] A. Kamath, O. Palmon, S. Plotkin, Routing and admission control in general topology networks with Poisson arrivals, J. Algorithms 27 (2) (1998) 236-258.

[15] D. Karger, S. Plotkin, Adding multiple cost constraints to combinatorial optimization problems, with applications to multicommodity flows, in: Proc. 27th Annual ACM Symposium on Theory of Computing, May 1995, pp. 18-25.

[16] F.P. Kelly, Blocking probabilities in large circuit-switched networks, Adv. in Appl. Prob. 18 (1986) $473-505$.

[17] P. Klein, S. Plotkin, C. Stein, É. Tardos, Faster approximation algorithms for the unit capacity concurrent flow problem with applications to routing and finding sparse cuts, SIAM J. Comput. 23 (3) (1994) 466-487.

[18] J. Kleinberg, E. Tardos, Disjoint paths in densely embedded graphs, in: 36th IEEE Symposium on Foundations of Computer Science, 1995, pp. 52-61.

[19] T. Leighton, F. Makedon, S. Plotkin, C. Stein, É. Tardos, S. Tragoudas, Fast approximation algorithms for multicommodity flow problems, J. Comput. System Sci. 50 (2) (1995) 228-243.

[20] Y. Ma, S. Plotkin, Improved lower bounds for load balancing of tasks with unknown duration, Inform. Process. Lett. 62 (6) (1997) 301-303.

[21] S. Plotkin, D. Shmoys, É. Tardos, Fast approximation algorithms for fractional packing and covering problems, Math. Oper. Res. 20 (2) (1995) 257-301.

[22] D. Sleator, R. Tarjan, Amortized efficiency of list update and paging rules, Comm. ACM 28 (2) (1985) 202-208. 